\title{
Review Article \\ Role of the Hedgehog Signaling Pathway in Regulating the Behavior of Germline Stem Cells
}

\author{
Shiqin Li, Meng Wang, Yanghui Chen, Wei Wang, Junying Wu, Chengpeng Yu, \\ Yuehui Zheng, and Zezheng Pan
}

Jiangxi Medical College, Nanchang University, Nanchang, China

Correspondence should be addressed to Zezheng Pan; panzz@ncu.edu.cn

Received 26 January 2017; Revised 11 May 2017; Accepted 21 May 2017; Published 13 August 2017

Academic Editor: Leonard M. Eisenberg

Copyright $@ 2017$ Shiqin Li et al. This is an open access article distributed under the Creative Commons Attribution License, which permits unrestricted use, distribution, and reproduction in any medium, provided the original work is properly cited.

\begin{abstract}
Germline stem cells (GSCs) are adult stem cells that are responsible for the production of gametes and include spermatogonial stem cells (SSCs) and ovarian germline stem cells (OGSCs). GSCs are located in a specialized microenvironment in the gonads called the niche. Many recent studies have demonstrated that multiple signals in the niche jointly regulate the proliferation and differentiation of GSCs, which is of significance for reproductive function. Previous studies have demonstrated that the hedgehog (Hh) signaling pathway participates in the proliferation and differentiation of various stem cells, including GSCs in Drosophila and male mammals. Furthermore, the discovery of mammalian OGSCs challenged the traditional opinion that the number of primary follicles is fixed in postnatal mammals, which is of significance for the reproductive ability of female mammals and the treatment of diseases related to germ cells. Meanwhile, it still remains to be determined whether the Hh signaling pathway participates in the regulation of the behavior of OGSCs. Herein, we review the current research on the role of the Hh signaling pathway in mediating the behavior of GSCs. In addition, some suggestions for future research are proposed.
\end{abstract}

\section{Introduction}

Stem cells have many possible applications based on their ability for differentiation. At present, advances in regenerative treatments with stem cells are progressing dramatically, bringing hope for individuals with certain complicated and refractory diseases, such as maculopathy and nerve injury $[1,2]$. GSCs are adult stem cells responsible for the production of gametes. The proper maintenance of GSCs is regulated by various signals in the surrounding microenvironment (the niche). In recent years, the hedgehog ( $\mathrm{Hh}$ ) signaling pathway was identified as a relevant regulator of GSCs through its direct regulation of GSCs and indirect regulation of the niche surrounding GSCs to affect GSCs. Because of their ability for differentiation, GSCs have possible applications in reproductive medicine. Here, we review and, respectively, discuss the effect of the Hh signaling pathway on the proliferation and differentiation of SSCs in Drosophila and male mammals, the migration of OGSCs from the ovarian surface epithelium (OSE) to the ovary cortex, and the development of OGSCs in the ovary cortex of mammals.

\section{Hedgehog Signaling Pathway}

In 1980, when Nusslein-Volhard and Wieschaus were screening genes that affected the growth of fly larvae, they found that a certain mutated gene caused hedgehog-like spiked protrusions on the ventral side of Drosophila embryos; thus, the gene was named $H h$ [3]. Afterwards, three genes, $S h h, D h h$, and $I h h$, homologous to $H h$, were found in vertebrates. The proteins encoded by all of the above genes can activate the Hh signaling pathway, which can regulate the function of stem cells, repair damaged cellular tissue, and maintain cellular structure $[4,5]$.

The classical Hh signaling pathway is composed of the Hh ligand, a membrane protein receptor complex of patched (Ptc) and smoothened (Smo) proteins, nuclear transcription factors, and target genes. Hh encodes the Hh precursor protein, which turns into the Hh ligand protein after selfcleavage and palmitoylation. The Hh ligand protein acts on target cells through paracrine or autocrine modes of action with the help of the dispatched (Disp) protein $[6,7]$. 
Interference hedgehog (iHOG; CDO is homologous to it in mammals) and brother of iHOG (BOI; BOC is homologous to it in mammals) are two transmembrane proteins in target cells that can help to reinforce the interaction between $\mathrm{Hh}$ and Ptc $[8,9]$. In the absence of $\mathrm{Hh}$, Ptc represses the activity of Smo, which inhibits the transduction of Hh signaling via Smo. In contrast, when Hh binds with Ptc, the inhibition of Smo is relieved, which promotes the phosphorylation of the downstream complex $\mathrm{SuFu} / \mathrm{Ci} / \mathrm{Fu}$ (in nonmammals) or $\mathrm{SuFu} / \mathrm{Gli} / \mathrm{Kif}$ (in mammals) and releases the nuclear transcription factors $\mathrm{Ci}$ or Gli (there are three types of Gli factors in mammals: Gli1, Gli2, and Gli3). Subsequently, transcription factors enter the nucleus to directly regulate the transcription of target genes. In addition, it has been shown that $\mathrm{Hh}$ signaling transduction requires the participation of primary cilium.

Generally, nuclear transcription factors are divided into transcription activators (CiA/GliA) and transcription repressors (CiR/GliR) [10]. In mammals, the activities of the proteins Gli1 and Gli2 are similar, and they mainly function as a transcription factor as does GliA. However, Gli3 mostly functions as a transcription repressor as does GliR. In the absence of Hh ligand, Gli3 is restricted to a microtubule complex composed of Kif7 (kinesin family member 7), SuFu (suppressor of fused), CK1 (casein kinase 1), PKA (protein kinase $\mathrm{A}$ ), and Gsk $3 \beta$ (glycogen synthase kinase 3 beta) and is phosphorylated by PKA, GSK3 $\beta$, and CK1. Then, Gli3 is recognized by the $\mathrm{E} 3$ ubiquitin ligase $\beta \operatorname{TrCP}$ (beta-transducin repeat-containing proteins). Meanwhile, the transcriptional activation region at the C-terminal end is removed, and Gli3 becomes a transcription repressor (GliR), which enters the cell nucleus and inhibits the transcription of target genes. When the Hh ligand is present, activated Smo mediates the phosphorylation of SuFu/Gli/Kif7 complexes that release Gli (mainly Gli1), which blocks the hydrolysis process of the transcriptional activation region at the $\mathrm{C}$ terminal end. Then, the full-length Gli acts like GliA to promote the transcription of target genes. It has been proven that Hh, Gli1, Ptc, and Bmp are target genes for the classical Hh signaling pathway.

\section{Overview of Germline Stem Cells (GSCs)}

GSCs are restoring cells in the gonads that have the ability to differentiate into germ cells. They exist in a microenvironment (niche) in the gonads that contain somatic cells. These somatic cells cooperatively regulate the proliferation and differentiation of GSCs. GSCs are usually called spermatogonial stem cells (SSCs) in males [11] and ovarian germline stem cells (OGSCs) in females [12].

3.1. Spermatogonial Stem Cells (SSCs). SSCs in Drosophila gonads are located in a niche composed of SSCs, hub cells (HCs), and cyst stem cells (CySCs). In Drosophila, at the apex of the bilateral testicle, there is a cluster of HCs. One side of the cluster connects with the basement membrane of the testicle; the other side is the binding site for SSCs and CySCs. Usually, there are six to nine SSCs on one side of the testicle, and each SSC is isolated by two irregular-shaped CySCs.
During spermatogenesis, SSCs divide asymmetrically into two daughter cells. One cell adheres to HCs to maintain self-renewal, while the other separates from the HCs and forms a gonialblast (GB), which enters the differentiation stage. CySCs divide into two cyst cells, which surround the $\mathrm{GB}$ and regulate its proliferation and differentiation [13-15].

In 1994, SSCs were first discovered in mammals in the basement membrane of the mouse testis seminiferous tubule, and they account for $0.02 \%-0.03 \%$ of the total cells in the testis [16]. They are located in a niche that is mainly composed of SSCs, Sertoli cells (SCs), Leydig cells (LCs), peritubular myoid (PTM) cells, and other spermatogonia and spermatic cells in various differentiation phases. Spermatogonia in the testes are classified into three types, including type A, type intermediate, and type B. Type A can be subdivided into type A single (As), type A paired (Apr), and type A aligned (Aal). Among them, type As has the lowest differentiation degree and is currently widely recognized as SSCs $[11,17]$.

3.2. Ovarian Germline Stem Cells (OGSCs). OGSCs in Drosophila are located in a niche composed of cap cells (CpCs), terminal filament cells (TFCs), and escort cells (ECs). The niche is located in the apex of the oviduct in the bilateral germarium. In general, there are two or three OGSCs in each germarium. They adhere to $\mathrm{CpCs}$ through the E-calcium adhesion protein. OGSCs can asymmetrically divide into two daughter cells: one cell turns into a new OGSC and continues to adhere to $\mathrm{CpCs}$ while the other one separates itself from $\mathrm{CpCs}$ and proceeds to becoming an oocyte latterly $[18,19]$. Furthermore, Zou et al. [20] first segregated a type of cell from the postnatal mouse ovarian surface epithelium (OSE) in 2009. These isolated cells could coexpress the germ cell-specific marker Mvh and the stem cell markers OCT-4 and SSEA-1 $[21,22]$, and they are able to differentiate into oocyte-like cells in vitro. This type of cell is known as an OGSC. Then, in 2012, White et al. [23] further isolated mitotically active germ cells from the ovaries of reproductive-age women that could generate oocytes in vitro and in vivo. Afterwards, in 2014, Dunlop et al. [24] purified OGSCs from the adult and bovine ovarian cortex. Hence, OGSCs in mammals especially in humans probably have great significance for the reproductive ability of female mammals and treatment of diseases related to germ cells. However, OGSCs are present in small amounts in the ovary. Thus, further studies on the molecular regulatory mechanism of OGSC behavior are especially crucial.

\section{Effect of the Hedgehog Signaling Pathway on SSCs}

4.1. Effect of the Hedgehog Signaling Pathway on Drosophila SSCs. In Drosophila, the hedgehog signaling pathway regulates the proliferation and differentiation of SSCs via an indirect mechanism. Although SSCs are closely conglutinated with HCs, SSCs cannot express Ptc and Smo and fail to receive Hh signaling from HCs. Furthermore, the Hh ligand originating from $\mathrm{HCs}$ directly binds with Ptc in CySCs, which results in the upregulation of the transcription of target genes, including $H h$ and $B m p$ (bone morphogenetic 


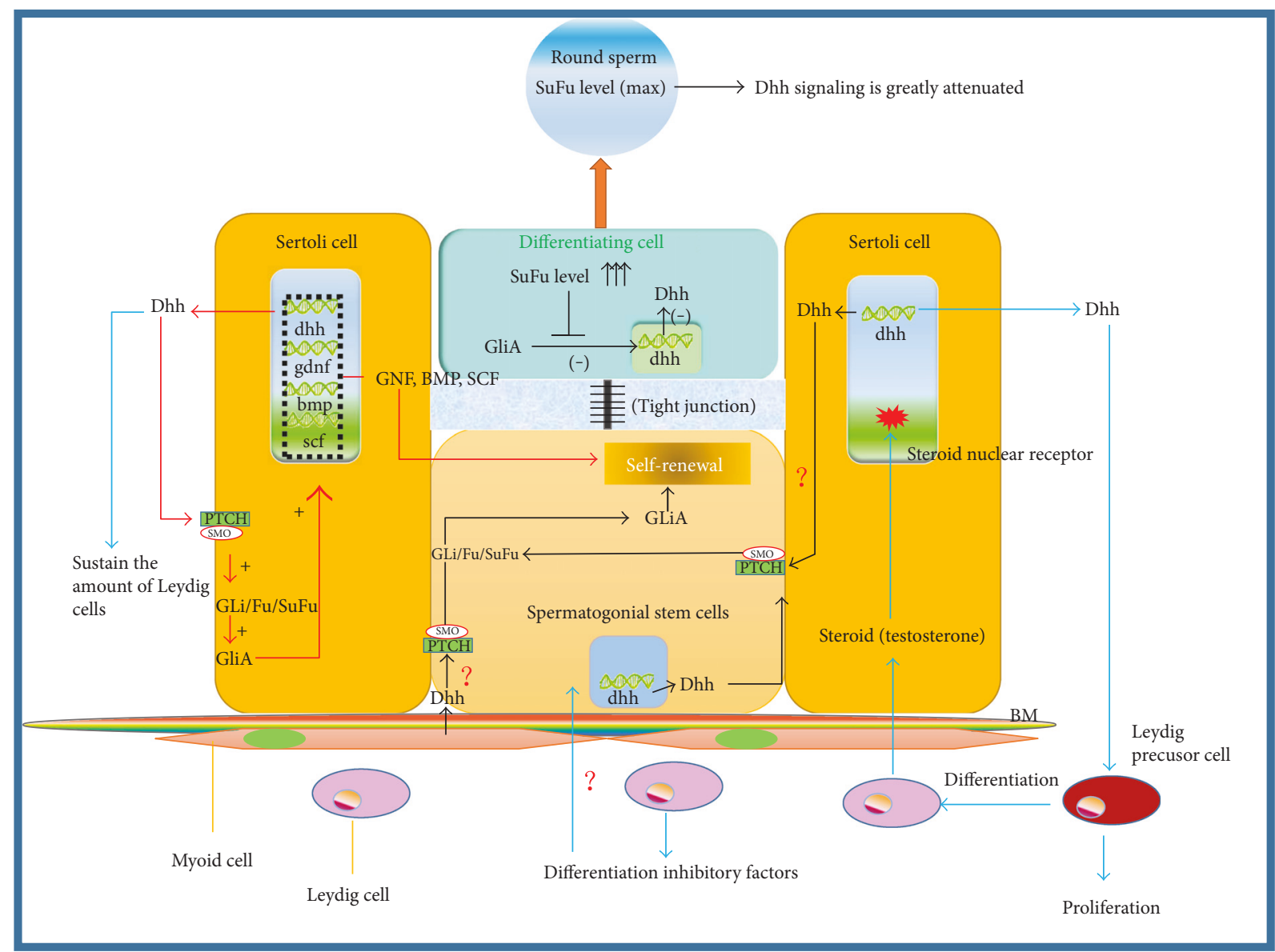

FIGURE 1: Effect of the Hh signaling pathway on the regulation of proliferation and differentiation of SSCs in mammals. (Arrowhead in red in this figure) Hh secreted by SCs can promote the expression of various factors in SCs, which helps to maintain SSC proliferation. (Arrowhead in blue in this figure) Dhh produced by SC is crucial for LCs for maintaining the number of LCs and expression of testosterone, which is favorable for the differentiation of SSCs. LCs also produce some undefined inhibitory factors that inhibit SSC differentiation. (Arrowhead in black in this figure) as SSCs differentiate, the expression of SuFu (a negative regulator of the Hh pathway) constantly increases. It inhibits the activity of the Hh pathway, which is beneficial for the differentiation of SSCs (arrowhead in black in this figure).

protein) [15]. The increased expression of Hh participates in the positive feedback of Hh signaling pathway activity in CySCs. Moreover, BMPs specifically activate the BMP signaling pathway in SSCs, which inhibits the transcription of bagof-marbles (Bam), which encodes Bam (differentiation-associated factor), to sustain the undifferentiated status of SSCs [25]. Hence, Hh signaling in the niche is an indirect signal that helps to sustain the proper number of SSCs. An overactivated or insufficiently activated $\mathrm{Hh}$ signaling pathway in CySCs would result in a decrease in SSCs. When the Hh signaling pathway is overactivated in CySCs, the number of SSCs sharply decreases probably because of the competition of abnormal proliferous CySCs. However, when the Hh signaling pathway is insufficiently activated, the number of CySCs decreases, and they fail to form the proper BMP concentration in the niche, which negatively affects the regeneration of SSCs [26]. Hence, controlling $\mathrm{Hh}$ signaling accurately in the SSC niche helps maintain SSCs, and it likely benefits the Drosophila spermatogenic cycle.

4.2. Hedgehog Signaling Pathway Regulates the Proliferation and Differentiation of Mammalian SSCs. In mammals, the
Hh signaling pathway regulates the proliferation and differentiation of SSCs in direct and indirect ways. Previous studies mainly considered that Hh signaling could indirectly affect the cellular behavior of SSCs through somatic cells in the niche. However, recent research revealed that SSCs could secrete Hh ligand and directly activate Hh signaling pathway in SSCs.

4.2.1. Hedgehog Signaling Pathway Indirectly Regulates the Proliferation and Differentiation of Mammalian SSCs. In mice, SSCs directly adhere to adult SCs in the niche. During the adult SC period, the transcription and sustained expression of Dhh can be detected in SCs. After secreting Dhh, adult SCs accept Hh signaling and promote the expression of glial cell-derived neurotrophic factor (GDNF), bone morphogenetic protein (BMP), and stem cell factor (SCF). These proteins can further activate other signaling pathways in SSCs, such as BMP, PI3K-Akt, Src, and RAS/ERK1/2, which inhibit the differentiation of SSCs and sustain a certain amount of SSCs [27-29] (Figure 1, arrowhead in red).

LCs and their precursor stem cells are located beneath the basement membrane of seminiferous tubules, and Ptc and 


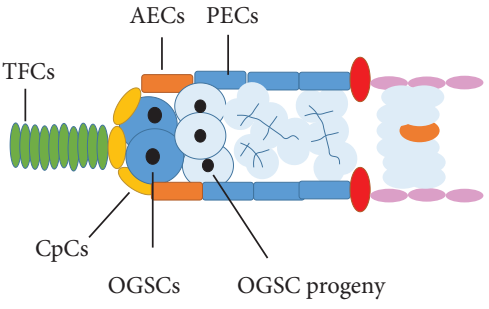

(a)

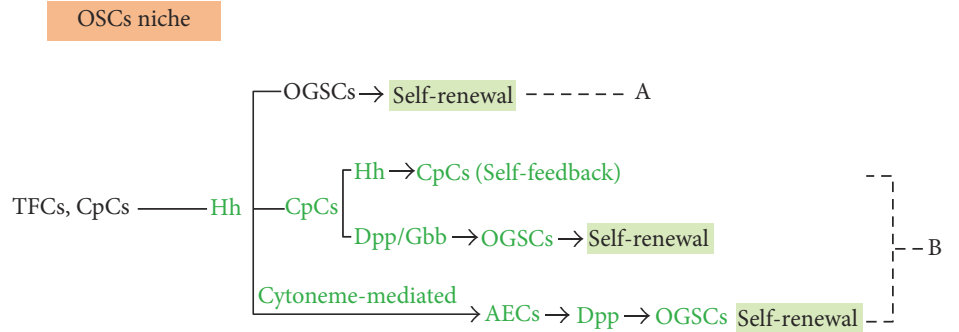

(b)

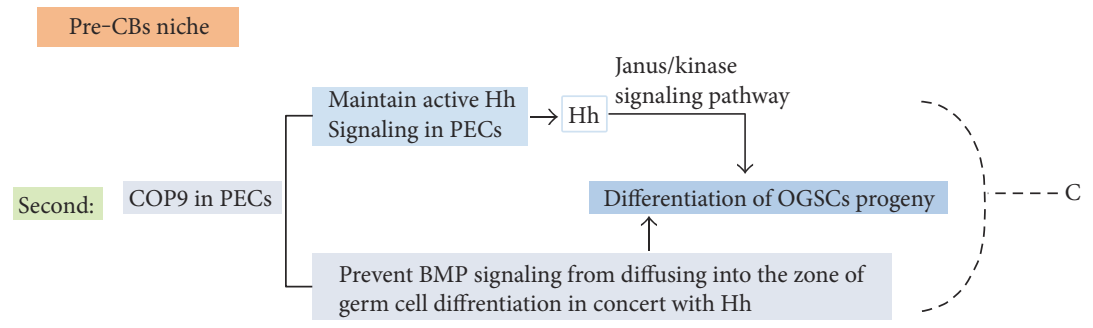

(c)

FIgURE 2: Effect of the Hh signaling pathway on the regulation of proliferation and differentiation of OGSCs in Drosophila. (a) OGSCs and their progeny are located in a niche that is composed of various somatic cells. (b) (A) Hh secreted from TFCs and CpCs can promote the self-renewal of OGSCs directly. (B) Hh originating from TFCs and CpCs can promote CpCs and AECs to secrete Gbb or Dpp, which is indirectly beneficial for the self-renewal of OGSCs. (c) (C) COP9 is important for maintaining the activity of the Hh pathway in PECs. The COP and $\mathrm{Hh}$ axis helps to prevent Dpp from diffusing. Moreover, COP9 can downregulate Dpp expression in PECs. A low concentration of Dpp antagonizes OGSC progeny differentiation.

Smo are on the surface of precursor stem cells. Dhh secreted by SCs affects the differentiation of Leydig lineage cells and establishment of the mature LC system, which is of great significance to the growth and development of SSCs [30, 31]. First, Dhh secreted by SCs is essential for the secretion of steroid hormones such as testosterone. Testosterone is indispensable in the process of spermatogenesis as it promotes the differentiation of SSCs into spermatids. Moreover, there are testosterone receptors in SCs. The hyposecretion of testosterone results in developmental defects in SCs, which negatively affect SSC differentiation [32]. Second, Dhh promotes the differentiation of LC precursor stem cells into adult LCs, and it is worth emphasizing that activated Hh signaling is a prerequisite for this differentiation process [33]. Third, research shows that there is primary cilium in poorly differentiated LCs in human adult testes [34]. However, the cilium will disappear when LCs develop into mature cells, which probably explains why LC precursor stem cells are sensitive to $\mathrm{Hh}$ signaling. Moreover, it also illustrates that Dhh secreted by SCs is important in establishing a mature LC system, which is ultimately favorable to SSC differentiation [35]. In addition, it has been reported that some undefined products seem to contribute to the inhibition of spermatogonial differentiation in irradiated rats [33]. Hence, during the process of SSC proliferation and differentiation, the balance between SCs and LCs regulated by Hh signaling is crucial [36] (Figure 1, arrowhead in blue).

4.2.2. Hedgehog Signaling Pathway Directly Regulates the Proliferation and Differentiation of Mammalian SSCs. In 2014, Sahin et al. [37] found that the transcription of the Hh pathway components Hh, Ptc, Smo, and Gli1 could be detected in an environment that solely contains undifferentiated SSCs. Moreover, SSCs did not differentiate and the number of SSCs increased in the first several months. Thus, they proposed that SSCs could maintain self-renewal before differentiation through an autocrine loop of $\mathrm{Hh}$ signaling. Meanwhile, SuFu is a negative regulatory factor in the $\mathrm{Hh}$ pathway, and it cannot be detected in earlier differentiation periods of SSCs. However, when SSCs develop into mature spermatids, the expression of SuFu constantly increases and inhibits Gli transcriptional activity, which results in the suppression of Dhh signaling in advanced circular sperm cells [38-40]. Therefore, as SSCs differentiate, their Hh signaling activity continuously decreases. The reason why $\mathrm{SuFu}$ is not expressed in earlier periods remains unknown. In addition, SSCs are the direct target cells of Hh ligand, which suggests that Dhh secreted by SCs perhaps could directly regulate the proliferation of SSCs. However, the regulatory effect of Dhh produced by SCs or PMCs on SSCs needs to be explored further (Figure 1, arrowhead in black).

\section{Effect of the Hedgehog Signaling Pathway on OGSCs}

5.1. Effect of the Hedgehog Signaling Pathway on Drosophila OGSCs. Hh signaling in the niche of OGSCs can suppress the differentiation of OGSCs in direct and indirect ways. Firstly, Hh signaling has been proposed to directly signal OGSCs and control the maintenance of OGSCs (Figure 2(b), A). Secondly, TFCs and CpCs are referred to as apical cells and can secret $\mathrm{Hh}$. The coreceptor of $\mathrm{Hh}$ iHOG/BOI can bind $\mathrm{Hh}$ with high affinity and sequester Hh on the surface, which inhibits Hh diffusion and forms a 
positive feedback of Hh pathway in apical cell. As target genes of Hh pathway, the expression of Hh increased [41, 42]. Then, CpCs deliver Hh signaling to AECs (ECs located in the anterior part of the germarium) and promote the transcription of the target genes Decapentaplegic (Dpp) and glass bottom boat (Gbb) (both belong to the Bmp family), in AECs $[27,43]$. Hh secreted by CpCs also directly enhances the transcription of $D p p$ in CpCs. Eventually, BMP (Dpp) signaling in the OGSCs niche is intensified. As a result, Dpp suppresses the expression of Bam and inhibits the differentiation of OGSCs. As OGSCs differentiate, the expression of Fused $(\mathrm{Fu})$, a positive regulator in the Hh pathway, is constantly increased. Moreover, Fu mediates the ubiquitination and proteolysis of thickveins (Tkv, a BMP receptor) in OGSCs, which is beneficial for the differentiation of OGSCs [19, 44-47] (Figure 2(b), B).

When OGSC progeny separates from CpCs, PEC- (ECs located in the posterior part of the germarium-) derived but not $\mathrm{CpC}$-derived $\mathrm{Hh}$ starts to participate in the differentiation process. It was discovered that maintaining the activation of the $\mathrm{Hh}$ signaling pathway and secretion of $\mathrm{Hh}$ ligand in PECs requires the presence of COP9 (also known as the CSN complex) in ECs [48]. Moreover, the COP9-Hh axis in PECs can partly prevent the diffusing of Dpp and promote the differentiation of OGSC progeny. In addition, JAK/STAT signaling promotes Dpp expression, whereas Hh signaling from PECs suppresses Dpp expression by antagonizing JAK/STAT signaling, which favors the differentiation of OGSC progeny. Therefore, it seems that Hh protein in the niche promotes the differentiation of OGSC progeny via suppressing the transcription of $D p p$ in a non-Hh signaling mechanism [49] (Figure 2(c), C).

5.2. Effect of the Hedgehog Signaling Pathway on Mammalian OGSCs. OGSCs in mammals are located in a single layer of epithelial cells with tight junctions in the OSE. In almost all species, it is not possible to observe obvious OGSC division in the normal OSE, and most research concerning OGSC function involves OGSC transplantation experiments. Thus, it is more reasonable to discuss the effect of Hh signaling pathway on OGSCs in normal OSE or in the ovary cortex after transplantation [50-52]. Moreover, it will be interesting to determine whether artificially altering the activity of $\mathrm{Hh}$ pathway will benefit ovum regeneration.

5.2.1. Effect of the Hedgehog Signaling Pathway on the Migration of OGSCs in the OSE. We proposed a hypothesis that the special structure of the OSE restricts the activity of OGSCs, but Hh signaling could help OGSCs migrate into the ovary cortex. Under natural conditions, the outside of the OSE is covered by the peritoneum, and the underside of the OSE adjoins the ovary cortex [53-55]. However, there is a layer of dense-constructed ovary tunica albuginea between the OSE and the ovary cortex. Restricted by this physiological structure, OGSCs seem to be isolated in an area that is distant from the ovary cortex, which contains various somatic cells such as granulosa cells (GCs), theca cells (TCs), and mesenchymal cells (MCs). Thus, in the normal ovary, it is difficult to predict how Hh signaling regulates the proliferation, differentiation, or any other cellular behavior of OGSCs through other somatic cells. Epithelial cells in the OSE are equipped with both epithelial and mesenchymal phenotypes. They can undergo epithelial-mesenchymal transition (EMT), which changes the tight junctions between epithelial cells into looser junctions between mesenchymal cells. Previous studies revealed that $\mathrm{Hh}$ signaling pathway participates in EMT in other tissues [56-58]. However, Glil transcription is not observed in the OSE, seemingly indicating that $\mathrm{Hh}$ pathway is not activated in the OSE in the normal state. However, the Hh signaling pathway participates in the migration of epithelial ovarian cancer cells into the ovary cortex. This suggests that the Hh pathway is possibly related to the migration of OGSCs into the ovary cortex. Once OGSCs shift to the cortex, they enter into an entirely different environment that contains various biosignals that probably promote the proliferation or differentiation of OGSCs (Figure 3(b)).

5.2.2. Effect of the Hedgehog Signaling Pathway on the Proliferation and Differentiation of OGSCs. It is not clear whether somatic cells in the ovary cortex regulate the function of OGSCs through the Hh signaling pathway. During the initial forming period of the mammalian fetal ovary, the transcription levels of $H h, P t c$, and Gli are very low [59]. After birth, the primordial oocyte of the primary follicle secretes the differentiation factor GDF9 (growth differentiation factor 9) [60], inducing granulosa cells to secrete Dhh and Ihh. Then, Dhh and Ihh continue to induce progenitor TCs to differentiate into TCs to promote follicle development [61-64]. In the ovary, GCs are the main source of Hh protein, while Ptc is the most abundant on the surface of TCs. Ptc not only binds with $\mathrm{Hh}$ but also works in preventing $\mathrm{Hh}$ signaling from diffusing $[63,65]$. Hence, Ptc in TCs distributed around the outer sphere of the follicle seems to help prevent Hh from diffusing into nearby follicles or interstitial cells. Thus, when we injected OGSCs into the ovary cortex, they came into direct contact with the somatic cells (Figure 3(c)). In 2015, a study reported that cancer stem cells of ovary cancer are very likely a malignant transformed product of OGSCs [66]. Furthermore, a previous study found that there is a BMP4-Hh-positive feedback loop between CSCs (cancer stem cells) of ovary cancer and CAMACs (cancer-associated mesenchymal stem cells), which enhances the proliferation of CSCs. Meanwhile, CSCs produce $\mathrm{Hh}$ to activate the BMP signaling pathway in CAMSCs [67]. Then, the overexpression of BMP4 suppresses CSCs to differentiate, resulting in the overexpression of $\mathrm{Hh}$. Although CSCs can secret $\mathrm{Hh}$, it is not clear whether CSCs have the ability to secrete Hh before or after canceration. Until now, no study has mentioned whether OGSCs can produce Hh. Normal MSCs (mesenchymal stem cells) can also produce BMPs but at lower levels compared with CA-MACs, so it is unclear whether OGSCs in the normal ovarian stroma together with MACs form a positive feedback loop like the BMP4-Hh feedback loop between CSCs and CA-MACs and promote the proliferation of OGSCs (Figures 3(a) and 3(d)). Furthermore, Park et al. [68] found that BMP4 could promote the differentiation of OGSCs into 


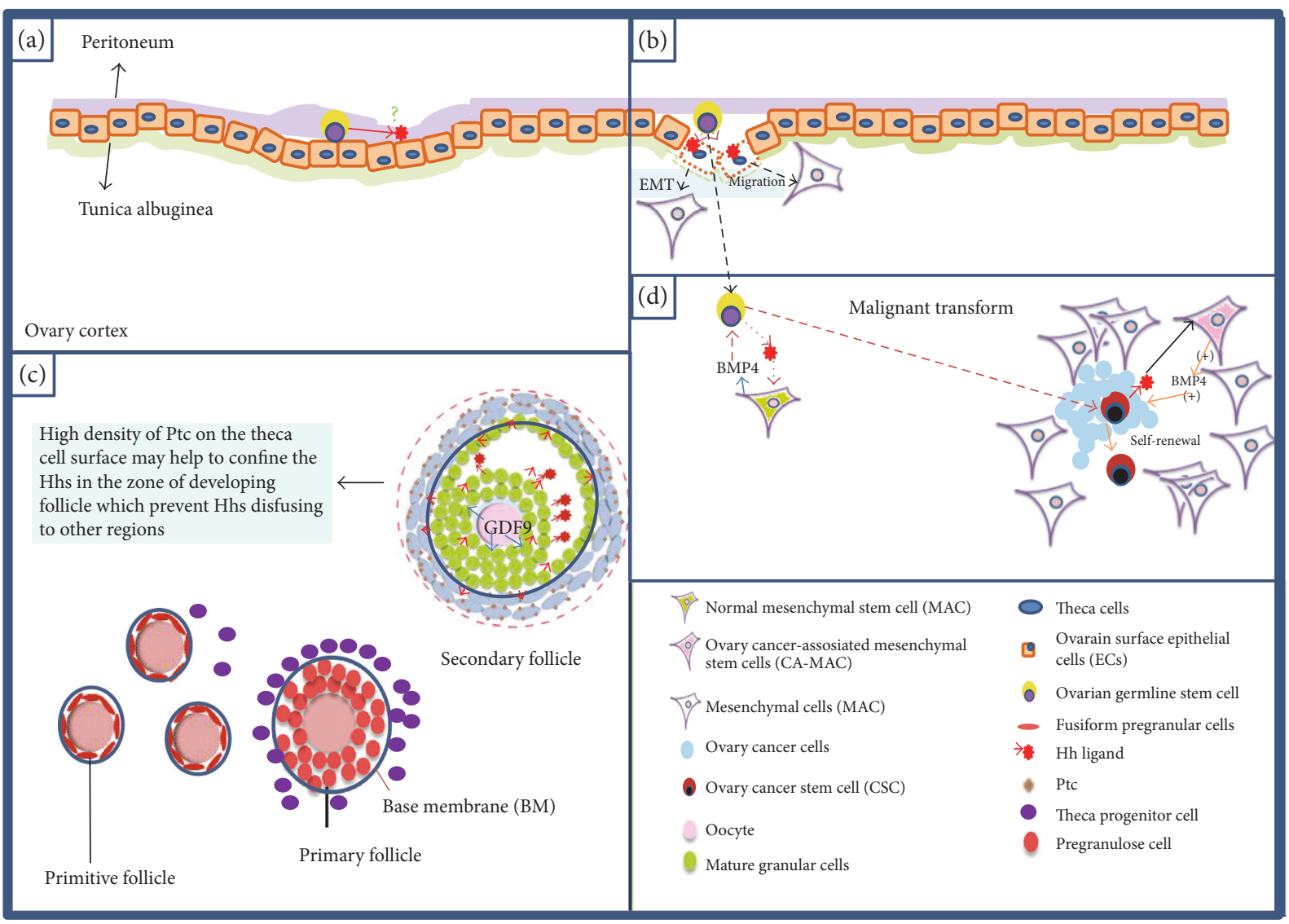

FIGURE 3: Effect of the Hh signaling pathway on the behavior of OGSCs in mammals. (a) It is not clear whether OGSCs in the ovarian surface epithelium (OSE) secrete Hh. (b) It is thought that the EMT could help OGSCs migrate to the ovary cortex from the OSE. (c) Ptc in TCs assists in preventing the Hh ligand from diffusing to other developing follicles and the interstitial stroma. (d) CSCs of ovarian cancer are likely to be the malignant transformation products of OGSCs. There is a BMP4-Hh feedback loop between CSCs and CA-MACs that promotes CSC proliferation and maintains the CSC number. It has not been determined whether there is a similar BMP4-Hh feedback loop between OGSCs and MSCs.

oocytes via Smad1/5/8 signaling in mice. This result was counter to the assumption that BMP4 helps to sustain the self-renewal of OGSCs. However, the result may depend on the dosage of BMP. Therefore, we conclude that the proliferation or differentiation of OGSCs varies with different concentrations of BMP4 conducted by $\mathrm{Hh}$ signaling from OGSCs. This resembles the dual regulation in Drosophila when $\mathrm{Hh}$ signaling stimulates other cells to secrete BMP protein.

Can somatic cells in the ovary regulate OGSC development as in Drosophila? Grieve et al. [51] found that stagespecific granulosa cells could induce the expression of oocyte-specific genes in embryonic stem cells under coculture conditions. He proposed that somatic cells are important for facilitating the differentiation of stem cells into functional oocytes. GCs can secrete the Hh ligand, but it is not clear whether the Hh pathway is involved in the proliferation and differentiation of OGSCs under coculture with OGSCs. However, it would perhaps be more convincing if we use OGSCs to replace the oogonia in primary follicles and then observe follicle development. A developing follicle is a good model for studying the relationship between GCs, Hh, and OGSCs. However, it is not clear whether Ptc and Smo are in OGSCs, which if so would indicate that altering Hh pathway activity would regulate the proliferation of OGSCs.

\section{Perspective}

According to the current research progress in this area, the following questions need to be thoroughly addressed in the near future.

Currently, many studies have indicated that the Hh signaling pathway has a strong effect on the proliferation and differentiation of SSCs. What we are mostly interested is whether we can artificially alter the self-renewal or differentiation process via regulating $\mathrm{Hh}$ signaling in the SSC niche, thereby improving the reproductive ability of animals. For example, some animals with azoospermia cannot produce a sufficient number of sperm and lose their reproductive ability because of the dysfunction of SSCs or the low number of SSCs. A sufficient number of SSCs is a prerequisite for a normal spermatogenic ability in males. In 2014, it was determined in testicular biopsies that the ectopic expression of Shh results in the absence of spermatocytes and increased numbers of LCs in the testes [69]. We discussed in this article that LCs have dual roles in regulating SSC physiology. Hence, 
the proper regulation of Hh signaling in the niche is vital to maintain the number of SSCs. In addition, apart from proliferation and differentiation, apoptosis is also an important physiological process of SSCs that could impact the number of SSCs. Moreover, the Hh signaling pathway possibly takes part in antiapoptosis, but there has been no relevant study. In Drosophila, the JAK-STAT signaling pathway, whose functions overlap with those of the Hh signaling pathway, enhances the proliferation of SSCs and the overexpression of the antiapoptosis protein DIAPI in cells, which helps sustain the activity of SSCs [70]. Thus, the Hh pathway probably functions in the antiapoptosis process. When SSCs differentiate, the increase in the SuFu level inhibits the activity of the $\mathrm{Hh}$ pathway. While there is little SuFu in SSCs, it is not known whether any substance other than SuFu antagonizes ectopic Hh signaling to maintain the proper number of SSCs. In short, regardless of what promotes the proliferation, differentiation, or antiapoptosis of SSCs via altering $\mathrm{Hh}$ signaling pathway activity, these results all provide some new ideas for clinical therapeutic methods for treating azoospermia or other diseases related to SSCs.

OGSCs are considered one of the possible cells in animals that can generate oocytes. Regulating OGSC function can be used to help those who wish to lengthen their reproductive life span or to treat animals or humans with germ cell dysfunction to restore their reproductive ability. Whether changing the Hh signaling pathway in OGSCs can affect the formation of primordial follicles in mammals and whether any effects on the proliferation and differentiation of OGSCs are caused by $\mathrm{Hh}$ signaling regulation still require further investigation. Previous studies have shown that Hh signaling can influence the development of TCs and hinder ovulation in mammals [71], but there has been no report on the effect of Hh signaling on OGSCs and primordial follicle formation. However, other signaling pathways, such as Hippo-YAP, Notch, and WNT [72-74], closely related to the Hh signaling pathway, have been found to be involved in regulating the proliferation and differentiation of OGSCs, as well as influencing the formation and development of mammalian primordial follicles. For example, Ci [75], a component of the Hh pathway, suppresses the activity of the Hippo signaling pathway kinase cascade in Drosophila ovary somatic cells, which eventually promotes the differentiation of OGSCs. Meanwhile, the Hippo-YAP signaling pathway has a negative regulatory effect on the generation and development of mammalian follicles [76], which probably indicates that the activation of the $\mathrm{Hh}$ pathway can enhance the growth and development of mammalian follicles via regulating the behavior of OGSCs.

\section{Conflicts of Interest}

The authors declare that there is no conflict of interests regarding the publication of this paper.

\section{Acknowledgments}

The authors thank all of the members in their lab. This work was supported by the National Natural Science Foundation of China (no. 81660245 and no. 81360100), the Excellence 555 Engineering of JiangXi Province, and the Natural Science Foundation of JiangXi Province (no. 20142BAB205069).

\section{References}

[1] P. Song, Y. Inagaki, Y. Sugawara, and N. Kokudo, "Perspectives on human clinical trials of therapies using iPS cells in Japan: reaching the forefront of stem-cell therapies," Bioscience Trends, vol. 7, no. 3, p. 157, 2013.

[2] A. S. Bryukhovetskiy and I. S. Bryukhovetskiy, "Effectiveness of repeated transplantations of hematopoietic stem cells in spinal cord injury," World Journal of Transplantation, vol. 5, no. 3, pp. 110-128, 2015.

[3] C. Nusslein-Volhard and E. Wieschaus, "Mutations affecting segment number and polarity in Drosophila," Nature, vol. 287, no. 30, p. 795801, 1980.

[4] P. W. Ingham and A. P. McMahon, "Hedgehog signaling in animal development: paradigms and principles," Genes \& Development, vol. 15, no. 23, pp. 3059-3087, 2001.

[5] C.-c. Hui and S. Angers, "Gli proteins in development and disease," Annual Review of Cell and Developmental Biology, vol. 27, pp. 513-537, 2011.

[6] J. A. Buglino and M. D. Resh, "Hhat is a palmitoylacyltransferase with specificity for N-palmitoylation of sonic hedgehog," The Journal of Biological Chemistry, vol. 283, no. 32, pp. 22076-22088, 2008.

[7] T. Kawakami, T. Kawcak, Y. J. Li, W. Zhang, Y. Hu, and P. T. Chuang, "Mouse dispatched mutants fail to distribute hedgehog proteins and are defective in hedgehog signaling," Development, vol. 129, no. 18, pp. 5753-5765, 2002.

[8] T. Tenzen, B. L. Allen, F. Cole, J. S. Kang, R. S. Krauss, and A. P. McMahon, "The cell surface membrane proteins Cdo and Boc are components and targets of the hedgehog signaling pathway and feedback network in mice," Developmental Cell, vol. 10, no. 5, pp. 647-656, 2006.

[9] S. Yao, L. Lum, and P. Beachy, "The ihog cell-surface proteins bind hedgehog and mediate pathway activation," Cell, vol. 125, no. 2, pp. 343-357, 2006.

[10] C. C. Hui and S. Angers, "Gli proteins in development and disease," Annual Review of Cell and Developmental Biology, vol. 27, p. 513537, 2011.

[11] S. S. Ram, B. T. Ozanna, C. Chhavi, and S. X. Hou, "Spermatogonial stem cells, infertility and testicular cancer," Journal of Cellular \& Molecular Medicine, vol. 15, no. 3, pp. 468-483, 2011.

[12] Z. Pan, M. Sun, X. Liang et al., "The controversy, challenges, and potential benefits of putative female germline stem cells research in mammals," Stem Cells International, vol. 2016, Article ID 1728278, 2016.

[13] L. J. Greenspan, M. D. Cuevas, and E. Matunis, "Genetics of gonadal stem cell renewal," Annual Review of Cell \& Developmental Biology, vol. 31, no. 31, p. 291, 2015.

[14] A. Spradling, M. T. Fuller, R. E. Braun, and S. Yoshida, "Germline stem cells," Cold Spring Harbor Perspectives in Biology, vol. 3, no. 3, article 002642, 2011.

[15] E. L. Matunis, R. R. Stine, and C. M. De, "Recent advances in Drosophila male germline stem cell biology," Spermatogenesis, vol. 2, no. 3, pp. 137-144, 2012.

[16] R. L. Brinster and J. W. Zimmermann, "Spermatogenesis following male germ-cell transplantation," Proceedings of the 
National Academy of Sciences of the United States of America, vol. 91, no. 24, pp. 11298-11302, 1994.

[17] Z. Chen, Z. Li, and Z. He, "Plasticity of male germline stem cells and their applications in reproductive and regenerative medicine," Asian Journal of Andrology, vol. 17, no. 3, pp. 367-372, 2014.

[18] F. J. King, A. Szakmary, D. N. Cox, and H. Lin, "Yb, modulates the divisions of both germline and somatic stem cells through piwi-, and hh- mediated mechanisms in the Drosophila ovary," Molecular Cell, vol. 7, no. 3, pp. 497-508, 2001.

[19] L. Xia, X. Zheng, W. Zheng et al., "The niche-dependent feedback loop generates a BMP activity gradient to determine the germline stem cell fate," Current Biology, vol. 22, no. 6, pp. 515-521, 2012.

[20] K. Zou, Z. Yuan, Z. Yang et al., "Production of offspring from a germline stem cell line derived from neonatal ovaries," Nature Cell Biology, vol. 11, no. 5, pp. 631-636, 2009.

[21] Z. Pan, M. Sun, J. Li et al., "The expression of markers related to ovarian germline stem cells in the mouse ovarian surface epithelium and the correlation with Notch signaling pathway," Cellular Physiology \& Biochemistry, vol. 37, no. 6, pp. 23112322, 2015.

[22] S. Parvari, M. Abbasi, N. Abbasi et al., "Stem cell isolation by a morphology-based selection method in postnatal mouse ovary," Archives of Medical Science, vol. 11, no. 3, pp. 670678, 2015.

[23] Y. A. White, D. C. Woods, Y. Takai, O. Ishihara, H. Seki, and J. L. Tilly, "Oocyte formation by mitotically active germ cells purified from ovaries of reproductive-age women," Nature Medicine, vol. 18, no. 3, pp. 413-421, 2012.

[24] C. E. Dunlop, R. A. Bayne, M. McLaughlin, E. E. Telfer, and R. A. Anderson, "Isolation, purifi cation, and culture of oogonial stem cells from adult human and bovine ovarian cortex," Lancet, vol. 383, 2017.

[25] M. Michel, A. P. Kupinski, I. Raabe, and C. Bökel, "Hh signalling is essential for somatic stem cell maintenance in the Drosophila testis niche," Development, vol. 139, no. 15, p. 2663, 2012.

[26] Z. Zhang, C. Pan, and Y. Zhao, "Hedgehog in the Drosophila testis niche: what does it do there?" Protein \& Cell, vol. 4, no. 9, pp. 650-655, 2013.

[27] M. Inaba, Y. M. Yamashita, and M. Buszczak, "Keeping stem cells under control: new insights into the mechanisms that limit niche-stem cell signaling within the reproductive system," Molecular Reproduction \& Development, vol. 83, no. 8, pp. 675-683, 2016.

[28] X. Meng, M. Lindahl, M. E. Hyvönen et al., "Regulation of cell fate decision of undifferentiated spermatogonia by GDNF," Science, vol. 287, no. 5457, pp. 1489-1493, 2000.

[29] J. M. Oatley and R. L. Brinster, “The germline stem cell niche unit in mammalian testes," Physiological Reviews, vol. 92, no. 2, pp. 577-595, 2012.

[30] L. J. Martin, "Cell interactions and genetic regulation that contribute to testicular Leydig cell development and differentiation," Molecular Reproduction \& Development, vol. 83, no. 6, pp. 470-487, 2016.

[31] R. Hazra, M. Jimenez, R. Desai, D. J. Handelsman, and C. M. Allan, "Sertoli cell androgen receptor expression regulates temporal fetal and adult Leydig cell differentiation, function, and population size," Endocrinology, vol. 154, no. 9, p. 3410, 2013.
[32] S. Y. Park, M. Tong, and J. L. Jameson, "Distinct roles for steroidogenic factor 1 and desert hedgehog pathways in fetal and adult Leydig cell development," Endocrinology, vol. 148, no. 8, pp. 3704-3710, 2007.

[33] I. Barsoum and H. H. C. Yao, "Redundant and differential roles of transcription factors Gli1 and Gli2 in the development of mouse fetal Leydig Cells1," Biology of Reproduction, vol. 84, no. 5, pp. 894-899, 2011.

[34] M. B. Nygaard, K. Almstrup, L. Lindbæk, S. T. Christensen, and T. Svingen, "Cell context-specific expression of primary cilia in the human testis and ciliary coordination of hedgehog signalling in mouse Leydig cells," Scientific Reports, vol. 5, pp. 1-14, 2015.

[35] H. L. Franco and H. H. Yao, "Sex and hedgehog: roles of genes in the hedgehog signaling pathway in mammalian sexual differentiation," Chromosome Research, vol. 20, no. 1, pp. 247-258, 2012.

[36] G. Shetty, W. Zhou, C. C. Weng, S. H. Shao, and M. L. Meistrich, "Leydig cells contribute to the inhibition of spermatogonial differentiation after irradiation of the rat," Andrology, vol. 18, no. 3, pp. 479-491, 2005.

[37] Z. Sahin, A. Szczepny, M. L. EA et al., "Dynamic hedgehog signalling pathway activity in germline stem cells," Andrology, vol. 2, no. 2, p. 267, 2014.

[38] J. A. Mäkelä, V. Saario, S. Bourguiba-Hachemi et al., "Hedgehog signalling promotes germ cell survival in the rat testis," Reproduction, vol. 142, no. 5, pp. 711-721, 2011.

[39] T. L. Kroft, J. Patterson, J. Won Yoon et al., "GLI1 localization in the germinal epithelial cells alternates between cytoplasm and nucleus: upregulation in transgenic mice blocks spermatogenesis in pachytene," Biology of Reproduction, vol. 65, no. 6, p. 1663, 2001.

[40] A. Szczepny, G. R. Hime, and K. L. Loveland, "Expression of hedgehog signalling components in adult mouse testis," Developmental Dynamics, vol. 235, no. 11, p. 3063, 2006.

[41] T. R. Hartman, D. Zinshteyn, H. K. Schofield, E. Nicolas, A. Okada, and A. M. O'Reilly, "Drosophila Boi limits hedgehog levels to suppress follicle stem cell proliferation," Journal of Cell Biology, vol. 191, no. 5, pp. 943-952, 2010.

[42] T. R. Hartman, T. I. Strochlic, Y. Ji, D. Zinshteyn, and A. M. O'Reilly, "Diet controls Drosophila follicle stem cell proliferation via hedgehog sequestration and release," Journal of Cell Biology, vol. 201, no. 5, pp. 741-757, 2013.

[43] P. Rojasríos, I. Guerrero, and A. Gonzálezreyes, "Cytonememediated delivery of hedgehog regulates the expression of bone morphogenetic proteins to maintain germline stem cells in Drosophila," PLoS Biology, vol. 10, no. 4, article e1001298, 2012.

[44] W. Lu, M. O. Casanueva, A. P. Mahowald, M. Kato, D. Lauterbach, and E. L. Ferguson, "Niche-associated activation of rac promotes the asymmetric division of Drosophila female germline stem cells," PLoS Biology, vol. 10, no. 7, article e1001357, 2012.

[45] L. Xia, S. Jia, S. Huang et al., "The fused/Smurf complex controls the fate of Drosophila germline stem cells by generating a gradient BMP response," Cell, vol. 143, no. 6, p. 978, 2010.

[46] S. Eliazer and M. Buszczak, "Finding a niche: studies from the Drosophila ovary," Stem Cell Research \& Therapy, vol. 2, no. 6, pp. 1-8, 2011.

[47] K. Narbonne-Reveau, F. Besse, C. Lamour-Isnard, D. Busson, and A. M. Pret, "Fused, regulates germline cyst mitosis and 
differentiation during Drosophila, oogenesis," Mechanisms of Development, vol. 123, no. 3, pp. 197-209, 2006.

[48] T. Lu, S. Wang, Y. Gao et al., "COP9-hedgehog axis regulates the function of the germline stem cell progeny differentiation niche in the Drosophila ovary," Development, vol. 142, no. 24, pp. 4242-4252, 2015.

[49] Z. Liu, G. Zhong, P. C. Chai et al., "Coordinated nicheassociated signals promote germline homeostasis in the Drosophila ovary," Journal of Cell Biology, vol. 211, no. 2, p. 469, 2015.

[50] I. Virantklun, "Postnatal oogenesis in humans: a review of recent findings," Stem Cells \& Cloning Advances \& Applications, vol. 8, pp. 49-60, 2015.

[51] K. M. Grieve, M. McLaughlin, C. E. Dunlop, E. E. Telfer, and R. A. Anderson, "The controversial existence and functional potential of oogonial stem cells," Maturitas, vol. 82, no. 3, p. 278, 2015.

[52] O. Celik, E. Celik, I. Turkcuoglu, E. Yilmaz, Y. Simsek, and B. Tiras, "Germline cells in ovarian surface epithelium of mammalians: a promising notion," Reproductive Biology and Endocrinology, vol. 10, no. 1, pp. 1-8, 2012.

[53] W. J. Murdoch and A. C. Mcdonnel, "Roles of the ovarian surface epithelium in ovulation and carcinogenesis," Reproduction, vol. 123, no. 123, pp. 743-750, 2002.

[54] E. Lengyel, "Ovarian cancer development and metastasis," American Journal of Pathology, vol. 177, no. 3, pp. 10531064, 2010.

[55] U. Urzúa, S. Ampuero, K. F. Roby, G. A. Owens, and D. J. Munroe, "Dysregulation of mitotic machinery genes precedes genome instability during spontaneous pre-malignant transformation of mouse ovarian surface epithelial cells," $B M C$ Genomics, vol. 17, no. 8, p. 728, 2016.

[56] S. Lamouille, J. Xu, and R. Derynck, "Molecular mechanisms of epithelial-mesenchymal transition," Nature Reviews Molecular Cell Biology, vol. 15, no. 3, pp. 178-196, 2014.

[57] A. Omenetti, A. Porrello, Y. Jung et al., "Hedgehog signaling regulates epithelial-mesenchymal transition during biliary fibrosis in rodents and humans," Journal of Clinical Investigation, vol. 118, no. 10, pp. 3331-3342, 2008.

[58] B. Davidson, C. G. Tropé, and R. Reich, "Epithelial-mesenchymal transition in ovarian carcinoma," Frontiers in Oncology, vol. 2, p. 33, 2012.

[59] N. C. Bingham, K. L. Parker, J. S. Jorgensen, and H. H. Yao, "Activation of the hedgehog pathway in the mouse fetal ovary leads to ectopic appearance of fetal Leydig cells and female pseudohermaphroditism," Developmental Biology, vol. 329, no. 1, p. 96, 2009.

[60] F. C. de Castro, M. H. Cruz, and C. L. Leal, "Role of growth differentiation factor 9 and bone morphogenetic. Protein 15 in ovarian function and their importance in mammalian female fertility-a review," Asian-Australasian Journal of Animal Sciences, vol. 29, no. 8, pp. 1065-1074, 2016.

[61] M. E. Pepling, "Hedgehog signaling in follicle development," Biology of Reproduction, vol. 86, no. 6, p. 173, 2012.

[62] L. J. Spicer, S. Sudo, P. Y. Aad et al., "The hedgehog-patched signaling pathway and function in the mammalian ovary: a novel role for hedgehog proteins in stimulating proliferation and steroidogenesis of theca cells," Reproduction, vol. 138, no. 2, pp. 329-339, 2009.
[63] M. C. Russell, R. G. Cowan, R. M. Harman, A. L. Walker, and S. M. Quirk, "The hedgehog signaling pathway in the mouse ovary," Biology of Reproduction, vol. 77, no. 2, p. 226, 2007.

[64] Y. Ren, R. G. Cowan, F. F. Migone, and S. M. Quirk, "Overactivation of hedgehog signaling alters development of the ovarian vasculature in mice," Biology of Reproduction, vol. 86, no. 6 , p. 174, 2012.

[65] Y. Chen and G. Struhl, "Dual roles for patched in sequestering and transducing hedgehog," Cell, vol. 87, no. 87, pp. 553-563, 1996.

[66] O. B. Ozakpinar, A. M. Maurer, and D. Ozsavci, "Ovarian stem cells: From basic to clinical applications," World Journal of Stem Cells, vol. 7, no. 4, pp. 757-768, 2015.

[67] L. G. Coffman, Y. J. Choi, K. McLean, B. L. Allen, M. P. di Magliano, and R. J. Buckanovich, "Human carcinomaassociated mesenchymal stem cells promote ovarian cancer chemotherapy resistanceviaa BMP4/HH signaling loop," Oncotarget, vol. 7, no. 6, pp. 6916-6932, 2016.

[68] E. S. Park, D. C. Woods, and J. L. Tilly, "Bone morphogenetic protein 4 (BMP4) promotes mammalian oogonial stem cell differentiation via Smad1/5/8 signaling," Fertility \& Sterility, vol. 100, no. 5, p. 1468, 2013.

[69] S. Zou, Y. Wang, T. Chen et al., "Ectopic expression of sonic hedgehog in a cryptorchid man with azoospermia: a case report," The Journal of International Medical Research, vol. 42, no. 2, pp. 589-597, 2014.

[70] S. Hasan, P. Hétié, and E. L. Matunis, "Niche signaling promotes stem cell survival in the Drosophila testis via the JAK-STAT target DIAP1," Developmental Biology, vol. 404, no. 1, pp. 27-39, 2015.

[71] Y. Ren, R. G. Cowan, R. M. Harman, and S. M. Quirk, "Dominant activation of the hedgehog signaling pathway in the ovary alters theca development and prevents ovulation," Molecular Endocrinology, vol. 23, no. 5, p. 711, 2009.

[72] S. Klusza and W. M. Deng, "At the crossroads of differentiation and proliferation: precise control of cell-cycle changes by multiple signaling pathways in Drosophila follicle cells," BioEssays, vol. 33, no. 2, pp. 124-134, 2011.

[73] C. P. Zhang, J. L. Yang, J. Zhang et al., "Notch signaling is involved in ovarian follicle development by regulating granul-osa cell proliferation," Endocrinology, vol. 152, no. 6, pp. 2437-2447, 2011.

[74] C. L. Chen, X. F. Fu, L. Q. Wang et al., "Primordial follicle assembly was regulated by Notch signaling pathway in the mice," Molecular Biology Reports, vol. 41, no. 3, pp. 18911899, 2014.

[75] C. Li, L. Kan, Y. Chen et al., "Ci antagonizes Hippo signaling in the somatic cells of the ovary to drive germline stem cell differentiation," Cell Research, vol. 25, no. 10, p. 1152, 2015.

[76] K. Kawamura, Y. Cheng, N. Suzuki et al., "Hippo signaling disruption and Akt stimulation of ovarian follicles for infertility treatment," Proceedings of the National Academy of Sciences of the United States of America, vol. 110, no. 43, pp. 1747417479, 2013. 

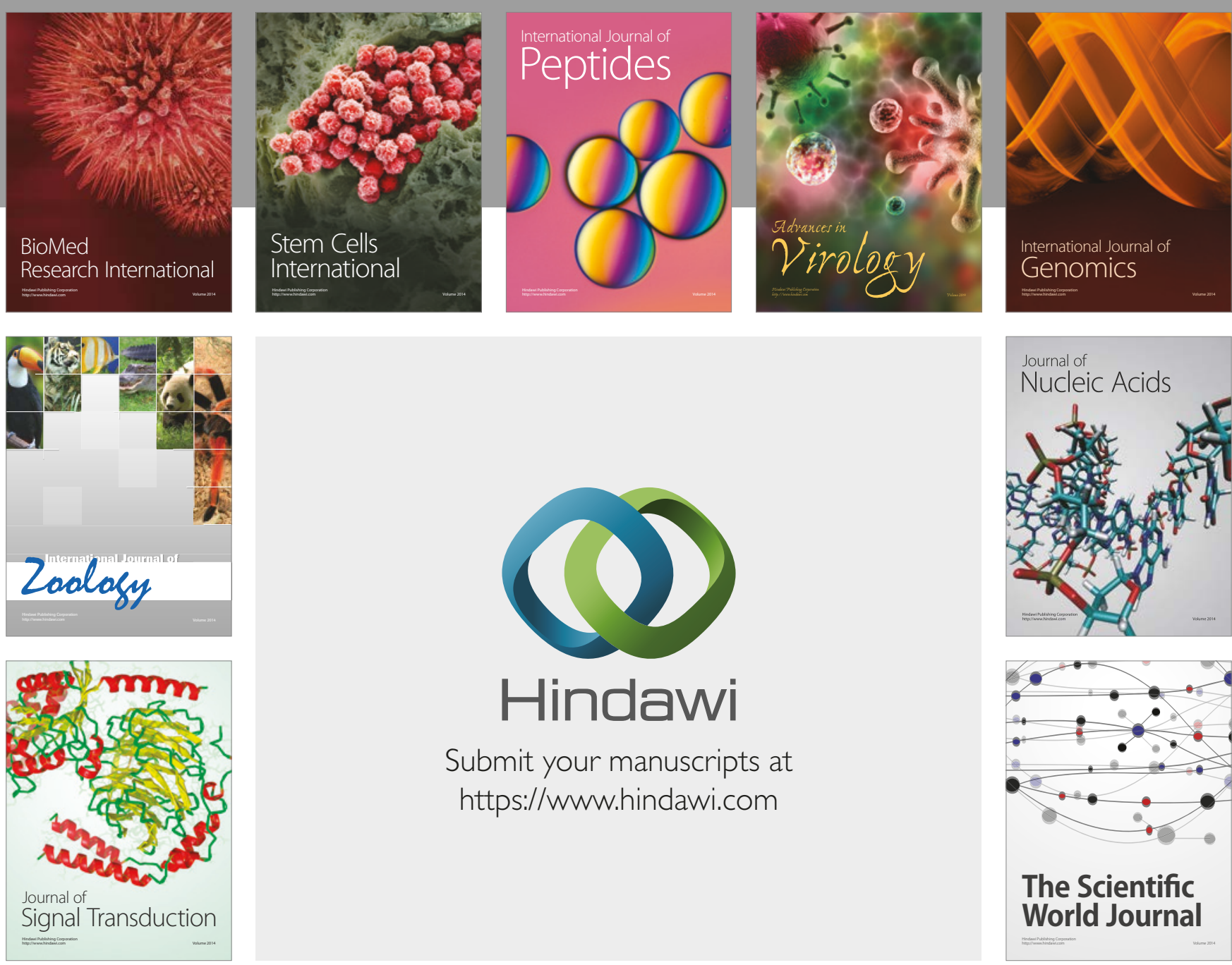

Submit your manuscripts at

https://www.hindawi.com
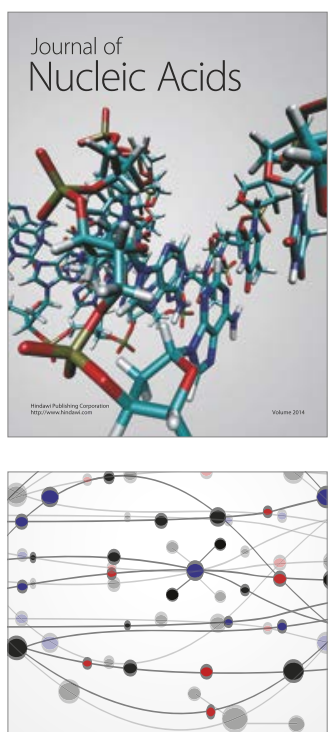

The Scientific World Journal

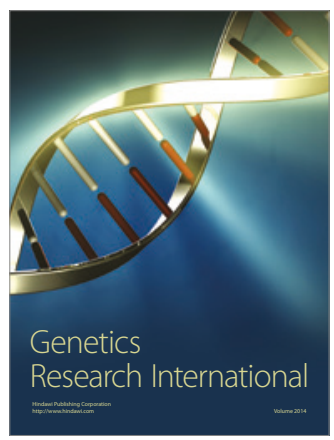

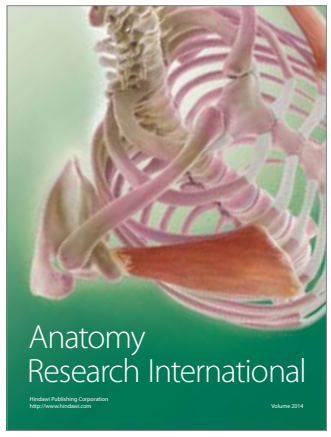

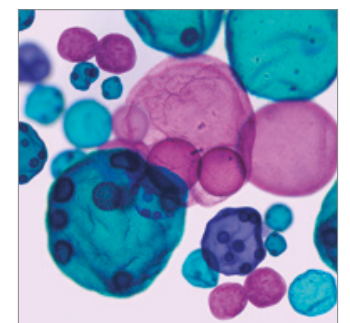

International Journal of Microbiology
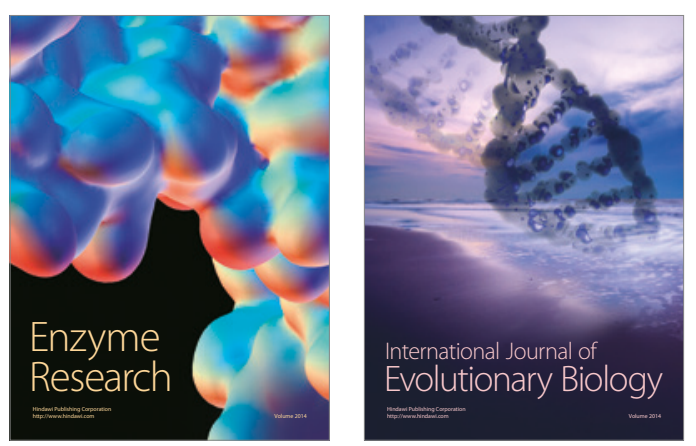
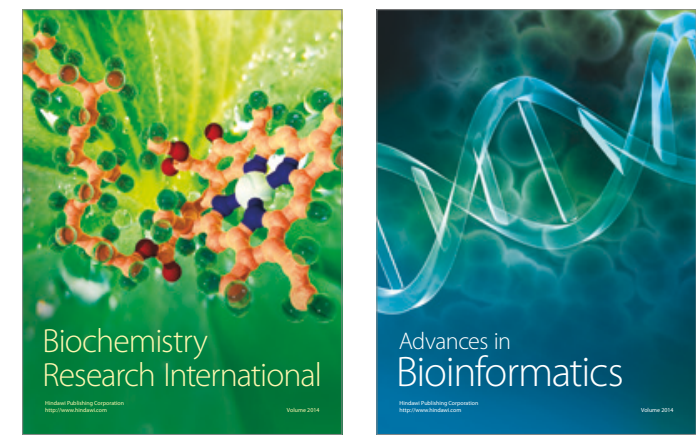

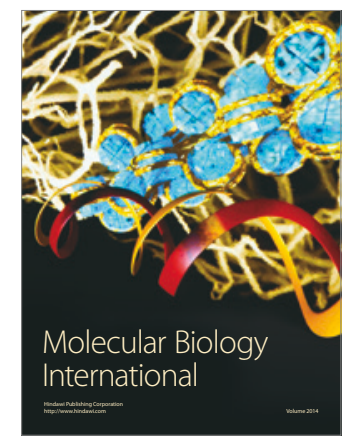

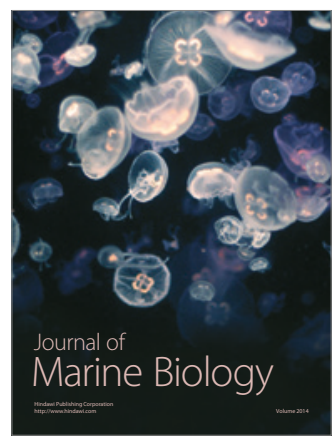

